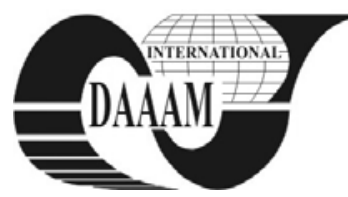

Annals of DAAAM for 2011 \& Proceedings of the 22nd International DAAAM Symposium, Volume 22, No. 1, ISSN 1726-9679 ISBN 978-3-901509-83-4, Editor B. Katalinic, Published by DAAAM International, Vienna, Austria, EU, 2011 Make Harmony between Technology and Nature, and Your Mind will Fly Free as a Bird Annals \& Proceedings of DAAAM International 2011

\title{
VEHICLE BEAM LIGHT ASSISTANT SYSTEM
}

\author{
ZENZEROVIC, P[aolo] \& CAR, Z[latan]
}

\begin{abstract}
This paper presents a system for automatic beam light detection from oncoming vehicles traveling in the opposite direction to the vehicle in which the system is implemented. The system is designed to operate during low light conditions (at night), with the goal to automatically change light intensity from high beam to low beam when a vehicle is detected. An approach using artificial neural network for image classification is proposed and implemented and test results are given in the paper. Furthermore, directions for future work and conclusions are presented.
\end{abstract}

Key words: neural network, beamlight, vehicle, driver assistance

\section{INTRODUCTION}

Good road illumination is one of the most important factors contributing to safe driving in low light conditions. Most vehicle producers use different lamps to provide the best possible lighting conditions for the vehicle drivers. Typically, headlamps can generate high and low intensity beams. The beams intensity is controlled by the driver and depends on the road drive conditions. High intensity beams provide more light to illuminate a wider and longer path in front of the vehicle, but High can only be used when there are no other vehicles on the road, because glare from the lights can affect other vehicle drivers. The effect of headlight glare has been studied over the past years (Akashi \& Rea, 2001).

To prevent this an automatic system for lowering the light beams can be used. The system proposed in this paper addresses only the problem of detecting oncoming vehicles coming in opposite direction to the vehicle in which the system is implemented. The complete solution should also include detection of tail lights on vehicles traveling in the same direction as the vehicle in which the system is implemented (Alcantarilla et al., 2008.). Other systems of vehicle detection have been proposed and discussed but none are yet used in massive serial production of vehicles (Alcantarilla et al., 2008.; Chen et al., 2006.; Gentex corporation; Lexus corporation).

Different methods for oncoming vehicle detection and headlight control have also been proposed by others. (Heinrich et al., 2009.; Toyota corporation, 2009.)

The main steps of solving this problem are discussed in the next section and experimental results are given in section III. In section IV a conclusion and ideas for future work are presented.

\section{IMPLEMENTED SYSTEM}

The system is composed of the following parts: acquisition camera, central processing unit and an actuator unit. The acquisition camera is mounted in the center of the windshield, inside the car. The implemented system uses a cheap USB camera, typically used for video chat etc. The central processing unit is a personal computer, running a program for image acquisition and processing, using artificial neural network for classification and a serial port based interface to switch from high to low beams. The written program completely runs under MATLAB programming suite. The camera captures images that approximate the drivers vision field. The images, which are 160x120 in size, are fed in the PC and then transformed from RGB (Red-Green-Blue) to grayscale format. Some samples of captured pictures are shown in Figure 1.

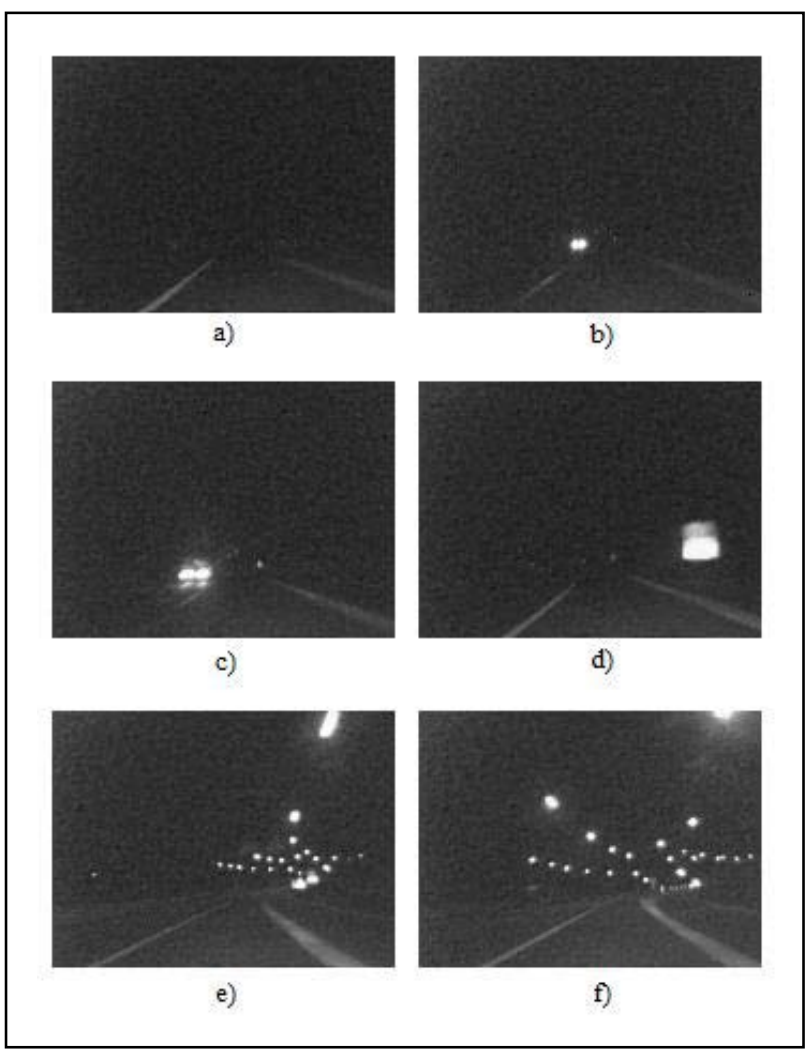

Fig. 1. Captured images samples

Figure 1a, 1b and 1c show typical situations, a road without incoming vehicles, an incoming vehicle in the distance and a close vehicle, respectively. Figures $1 \mathrm{~d}, 1 \mathrm{e}$ and $1 \mathrm{f}$ show images of captured road signs which reflect lights from the vehicle in which the system is implemented and glare from road lights. Image preprocessing with MATLAB Image Toolbox is used to eliminate all noise, using the thresholding method and geometry properties of the bright objects in the picture. The first criteria is to eliminate all bright spots which are smaller than a certain area. This eliminates background camera noise. The second problem is to eliminate road signs. This is done by segmentation of the image and inspection of the object excentricity. With this method square shaped road signs are eliminated. Then, the image is fed into the artificial neural network for classification.

The chosen network is a feedfoward network using a backpropagation algorithm in the learning process. For the network development MATLAB Pattern Recognition Tool was 
used which is part of the Artificial Neural Network Toolbox. The network consists of an input layer, one hidden layer and an output layer. The input layer has 19200 input neurons (the value representing every pixel of the preprocessed image is directly fed to one input neuron), the hidden layer consists of 10 neurons, and the output layer has 2 neurons representing two classes - high beam and low beam. The training was done with approximately 750 images. The results are shown in Figure 2.

As seen in the test confusion matrix in Figure 2 the tested system was able to correctly classify more than $97 \%$ of the images.

\section{EXPERIMENTAL RESULTS}

Additional tests were made in offline mode (with preacquisitioned images) with about 5000 images. The test results confirmed the initial results shown in Figure 2. The system was also tested while in real time operation using a MacBook Pro running at $2.4 \mathrm{GHz}$ and $4 \mathrm{~GB}$ of RAM memory. In average the system was able to capture and process 15 images per second which was enough for real time usage.

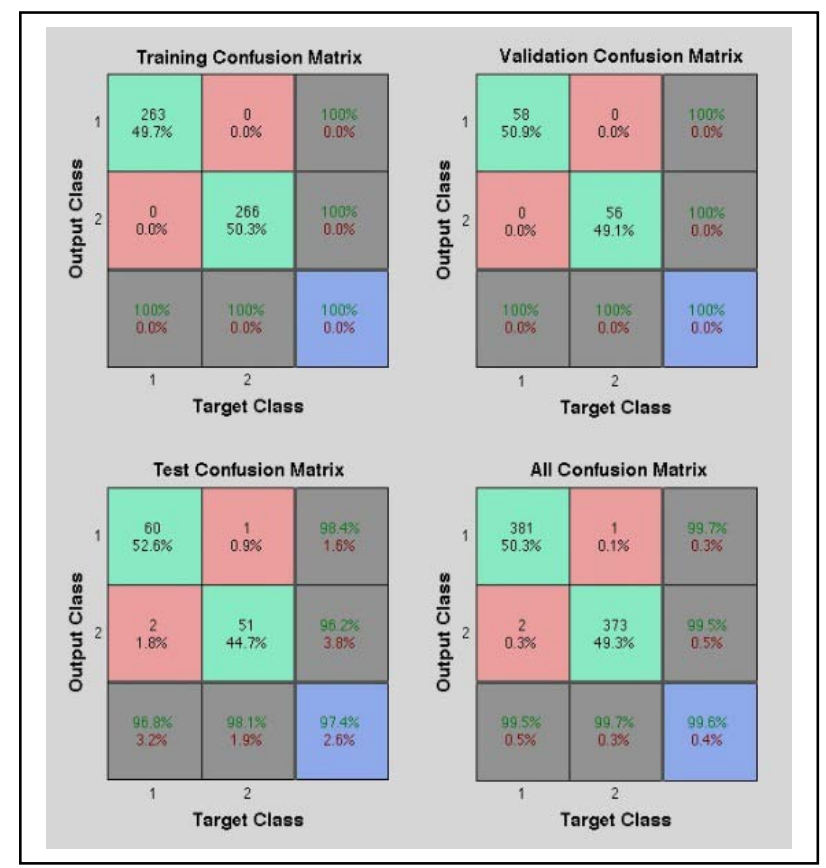

Fig. 2. Testing results

Also, tests were made to establish a minimal distance needed for a positive classification between the incoming vehicle and the vehicle in which the system is implemented. The results vary from 150 to 200 meters and depend on weather conditions, background lightening and vehicle beam intensity.

\section{CONCLUSION AND FUTURE WORK}

The paper presented a system for automatic detection of vehicle beam light at low light conditions (at night). The implemented system had good results in both the offline and real-time tests. On the other hand, the minimal distance needed for a positive classification between the incoming vehicle and the vehicle in which the system is implemented should be increased. Also, the system doesn't include tail light detection, which should be added in order to have a fully operational system intended implementation in vehicles. The authors plan to add this functionality to the system in the near future. Also, more image samples will be collected during different weather conditions to make the system robust for real life implementation.

\section{IMAGE DATABASE}

The image database used to train the artificial neural network and conduct tests of the implemented system was acquisitioned by the author of this paper. This process was time taking. Keeping this in mind, along with the need to have publically available databases for artificial neural network training, or general image processing the complete database will be posted and available online at (http://www.riteh.uniri.hr/zav_katd_sluz/zvd_pro_stroj/katedre/ kpor/nndatabase)

Other the the already mentioned image samples shown in Figure 1 additional usefull images for system testing can be found in the iomage database. Figure 3a and 3b show multiple road signs, Figure 3c shows multiple vehicles and Figure 3d shows vehicle tail lights.

Altogerther, about 10000 pictures will available in the image database.

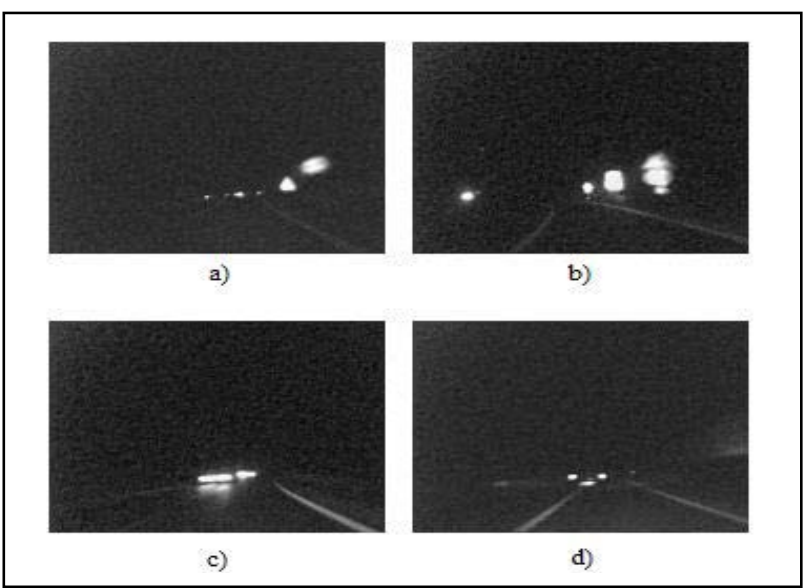

Fig. 3. Additional image samples

\section{ACKNOWLEDGEMENTS}

The authors appreciate the support of the CEEPUS (CIIIHR-0108) network in this project.

\section{REFERENCES}

Akashi, Y., \& Rea, M. (2001). The effect of oncoming headlight glare on peripheral detection under a mesopic light level, Available from: http://ken-gilbert.com/wrx/light s/PAL2001-akashi.pdf, Accessed on: 2011-8-15

Alcantarilla, P.F., Bergasa, L.M., Jimenez P., Sotelo M.A., Parra I. \& Fernandez D. (2008). Night time vehicle detection for driving assistance LightBeam Controller, Intelligent vehicle symposium, Eindhowen, ISSN: 19310587, ISBN: 978-1-4224-2568-6

Chen Y., Chen Y., Chen C. \& Wu B. (2006). Nighttime vehicle detection for driver assistance and autonomous vehicles, International Conference on Pattern Recognition, Hong Kong, ISSN: 1051-4661, ISBN: 0-7695-2521-0

Heinrich S., Fecher T., Almeida C. \& Kroekel D. (2009). Automatically adjusted headlamp beam, Available from: http://www.faqs.org/patents/app/20090296415, Accessed on: 2011-08-15

***Gentex corporation (2011), Light assistant, http://www.gentex.com/automotive/products/forwarddriving-assist, Accessed on: 2011-08-15

***Lexus corporation (2011), Automatic high beam manual, http://www.lexusmanuals.org/lexus-645.html, Accessed on: 2011-08-15

***Toyota corporation (2009), Intelligent adaptive frontlighting system, http://www.toyotaglobal.com/innovation/safety_technology_quality/safety_te chnology/technology_file/active/afs.html, Accessed on: 2011-08-15 